\title{
A new luminescent molecular based terbium hybrid material containing both organic polymeric chains and inorganic silica networks
}

\author{
Kai Sheng $\cdot$ Bing Yan
}

Received: 25 October 2008/Accepted: 10 March 2009/Published online: 21 March 2009

(C) The Author(s) 2009. This article is published with open access at Springerlink.com

\begin{abstract}
We have synthesized the ternary organic/ inorganic/polymeric terbium hybrid material (ACAC-Si)Tb-PVP $\quad[\mathrm{ACAC}=$ acetylacetone; $\mathrm{PVP}=$ poly $(4$-vinylpyridine)]. Firstly, the ACAC was modified through the hydrogen transfer addition reaction with a cross-linking reagent 3-(triethoxysilyl)-propyl isocyanate (TEPIC) to obtain a functional molecular bridge ACAC-Si which subsequently coordinated with $\mathrm{Tb}^{3+}$ and thus formed the inorganic Si-O-Si networks with TEOS (tetraethoxysilane) through hydrolysis and polycondensation process, and PVP acted as organic polymeric chains was synthesized using 4-vinylpyridine as the monomer after coordination with $\mathrm{Tb}(\mathrm{III})$ in the presence of BPO (Benzoyl Peroxide). For comparison, the binary hybrid material Tb-(ACAC-Si) was also prepared. The properties of the two hybrid materials were characterized in detail. The results reveal that the terbium complex was embedded into the organic polymer matrix by covalent bond with uniform microstructure. Furthermore, the distinction of the excitation spectrum between the binary and ternary hybrid materials suggests that the introduction of the polymeric organic chain component changed the microenvironment around the coordination sphere of the terbium. The emission spectrum exhibit the strong characteristic fluorescence of $\mathrm{Tb}^{3+}$, which accounts for the efficient intro-molecular energy transfer taken place in the two hybrid materials.
\end{abstract}

K. Sheng · B. Yan $(\bowtie)$

Department of Chemistry, Tongji University, 200092 Shanghai, China

e-mail: byan@tongji.edu.cn

\section{Introduction}

Luminescent rare earth complexes have attracted a great deal of attention in view of their unique luminescence properties and potential technological applications in many aspects such as displays, optical glasses, solid-state lasers and optical amplifiers [1-3]. It is well known that the lanthanide ions have characteristic emission bands because of the $\mathrm{f}-\mathrm{f}$ electron transition, which belongs to the forbidden transition, resulting in the relatively low absorption coefficient. Thereby, lanthanide ions were rarely used alone as luminescent component. Among the various ligands, organic ligands especially $\beta$-diketone are peerless and much more effective due to the large delocalization of the $\pi$ electrons which contribute to the strong absorption bands [4-6]. In the past decades, lanthanide complexes especially $\beta$-diketonates have been intensively studied due to their inherent extremely sharp emission peaks and high quantum efficiency [7].

However, there are some disadvantages of the pure lanthanide $\beta$-diketone complex, for example, poor photostability, thermal stability and mechanical strength. For practical applications, it is better to embed the luminescent lanthanide compounds into a host matrix [8-10], especially inorganic matrices or organic polymer matrices to circumvent the shortcomings. Two embedding methods were reported in many investigations. One is conventional method (called doping method), another is covalent method, that means connecting by covalent bonds. Using the first method, nevertheless, the clustering or quenching of the luminescent lanthanide centers resulting from the high vibration energy of the surrounding hydroxyl groups often occurs due to the incorporation with weak interactions (such as hydrogen bonding, van der waals force or electrostatic forces) between the luminescent complex and the host 
$[11,12]$. Thus, more and more attention has been paid to the rare earth (RE) polymer complexes (the covalent method) because they have advantages of both luminescence of RE- $\beta$-diketone chelate complex and simple synthesis of the polymer [13]. Our research group has focused on the covalent linking ligand of lanthanide complex to the inorganic Si-O network through different modified routes, including the modification of active amino group, hydroxyl groups and carboxyl groups with coupling agent, etc. [14-17]. These studies suggest that the thermal stabilities and photophysical properties were improved by the host.

In this paper, we report the lanthanide ion $\mathrm{Tb}^{3+}$ coordinated not only to the inorganic polymeric networks (Si-O-Si) through the modification of the ACAC using TEPIC but also with the organic polymeric chains $(\mathrm{C}-\mathrm{C})$ which was synthesized using the monomer 4-vinylpyridine (VP) as the original reagent. The ligand was successfully modified and the organic/inorganic/polymeric hybrid material was obtained. For the luminance properties and the microstructure, we compared the binary hybrid material $\mathrm{Tb}$-(ACAC-Si) with the ternary organic/inorganic hybrid material (ACAC-Si)-Tb-PVP in detail.

\section{Experimental}

\subsection{Starting materials}

Terbium nitrate were obtained by dissolving $\mathrm{Tb}_{4} \mathrm{O}_{7}$ in concentrated nitric acid. 3-(Triethoxysilyl)-propyl isocyanate (TEPIC) was provided by Lancaster Synthesis Ltd, and the tetraethoxysilane (TEOS) by Aldrich. All the other solvents were purified by common methods.

\subsection{The synthesis of the precursor ACAC-Si}

About $1 \mathrm{mmol}$ of acetylacetone (ACAC) was first dissolved in refluxing anhydrous tetrahydrofuran (THF) by stirring, and then $2 \mathrm{mmol}$ of $\mathrm{NaH}(0.08 \mathrm{~g}, 60 \%)$ was added into the solution. Two hours later, $2.5 \mathrm{mmol}(0.56 \mathrm{~g})$ of TEPIC was put into the solution dropwise. The whole mixture was refluxed at $65^{\circ} \mathrm{C}$ under argon atmosphere for $8 \mathrm{~h}$. After isolation and purification of the sample using a rotary vacuum evaporator, a yellow oil ACAC-Si was obtained. ${ }^{1} \mathrm{H}$ NMR $\left(\mathrm{CDCl}_{3}, 500 \mathrm{MHz}\right) \mathrm{C}_{25} \mathrm{H}_{50} \mathrm{O}_{10} \mathrm{~N}_{2} \mathrm{Si}_{2}: \delta 8.03(2 \mathrm{H}, \mathrm{bs}$, $\mathrm{NH}), 3.83\left(12 \mathrm{H}, \mathrm{q}, \mathrm{CH}_{2}(\mathrm{OEt})\right), 3.52(4 \mathrm{H}, \mathrm{t}), 2.23(6 \mathrm{H}, \mathrm{s}$, $\left.\mathrm{CH}_{3}\right), 1.82(4 \mathrm{H}, \mathrm{m}), 1.23\left(18 \mathrm{H}, \mathrm{t}, \mathrm{CH}_{3}(\mathrm{OEt})\right), 0.72(4 \mathrm{H}, \mathrm{t})$.

2.3 The synthesis of the binary molecular-based hybrid material $\mathrm{Tb}-(\mathrm{ACAC}-\mathrm{Si})$

The precursor ACAC-Si was dissolved in anhydrous ethanol. Then a stoichiometric amount of $\mathrm{Tb}\left(\mathrm{NO}_{3}\right)_{3} \cdot 6 \mathrm{H} 2 \mathrm{O}$ was added to the solution with stirring by drop. After $2 \mathrm{~h}$, a stoichiometric amount of TEOS and one drop of diluted hydrochloric acid were put into the solution to promote hydrolysis. The molar ratio of $\mathrm{Tb}\left(\mathrm{NO}_{3}\right)_{3} \cdot 6 \mathrm{H} 2 \mathrm{O} / \mathrm{ACAC}-\mathrm{Si} /$ TEOS $/ \mathrm{H}_{2} \mathrm{O}$ was $1: 3: 6: 24$. The final hybrid material I was collected after removing the solvent at $80^{\circ} \mathrm{C}$ in a few days and the predicted structure of $\mathrm{Tb}-(\mathrm{ACAC}-\mathrm{Si})$ was obtained as outlined in Fig. 1.

2.4 The synthesis of the ternary rare earth complex/ polymer hybrid (ACAC-Si)-Tb-PVP

The rare earth complex/polymer composite (ACAC-Si)-TbPVP was prepared as follows: the coordination procedure between the precursor ACAC-Si and the central $\mathrm{Tb}^{3+}$ ion was the same as the above process of $\mathrm{Tb}$-(ACAC-Si). The monomer 4-vinylpyridine $(0.3 \mathrm{mmol})$ was added into the mixture after the coordination process between $\mathrm{Tb}^{3+}$ and ACAC-Si completed. And then the Benzoyl Peroxide (BPO) was put into the refluxing mixture at $80^{\circ} \mathrm{C}$. After the addition polymerization reaction has completed, which lasted for $12 \mathrm{~h}$, a stoichiometric amount of TEOS and one drop of diluted hydrochloric acid were put into the solution at room temperature to promote hydrolysis. The mole ratio of $\mathrm{Tb}\left(\mathrm{NO}_{3}\right)_{3} \cdot 6 \mathrm{H} 2 \mathrm{O} / \mathrm{ACAC}-\mathrm{Si} / \mathrm{PVP} / \mathrm{TEOS} / \mathrm{H}_{2} \mathrm{O}$ was $1: 3: 1$ : 6:24. The hybrid material II was obtained after removing the solvent at $80^{\circ} \mathrm{C}$ in a few days. The predicted structure of (ACAC-Si)-Tb-PVP was obtained as outlined in Fig. 1.

\subsection{Physical measurements}

All measurements were performed at room temperature. Infrared spectra were recorded on a Nexus 912 AO446 FT-IR spectrophotometer. We mixed the compound with the dried potassium bromide $(\mathrm{KBr})$ and then pressed into pellets. The spectra were collected over the range 4,000$400 \mathrm{~cm}^{-1}$ by averaging 32 scans at a maximum resolution of $4 \mathrm{~cm}^{-1}$. ${ }^{1} \mathrm{H}$ NMR spectra were recorded in $\mathrm{CDCl}_{3}$ on a Bruker AVANCE-500 spectrometer with tetramethylsilane (TMS) as an internal reference. The Ultraviolet absorption spectra were carried out on an Agilent 8453 spectrophotometer and the ultraviolet-visible diffuse reflection spectra of the powder samples were recorded by a BWS003 spectrophotometer. X-ray powder diffraction patterns were recorded using a Rigaku D/max-rB diffractometer system equipped with a $\mathrm{Cu}$ anode in a $2 \theta$ range from $10^{\circ}$ to $70^{\circ}$. The fluorescence excitation and emission spectra were obtained on a RF-5301 spectrophotometer equipped with a stablespec-xenon lamp $(450 \mathrm{~W})$ as the light source. The microstructures were checked by scanning electronic microscopy (SEM, Philip XL-30). 


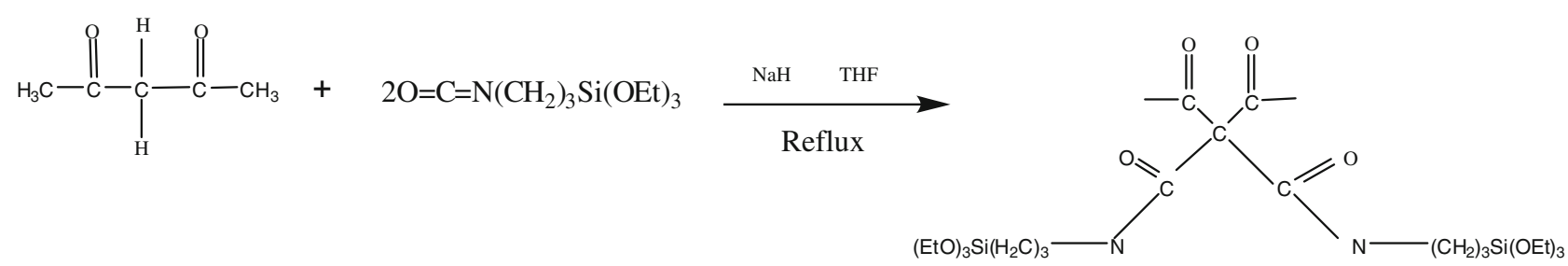

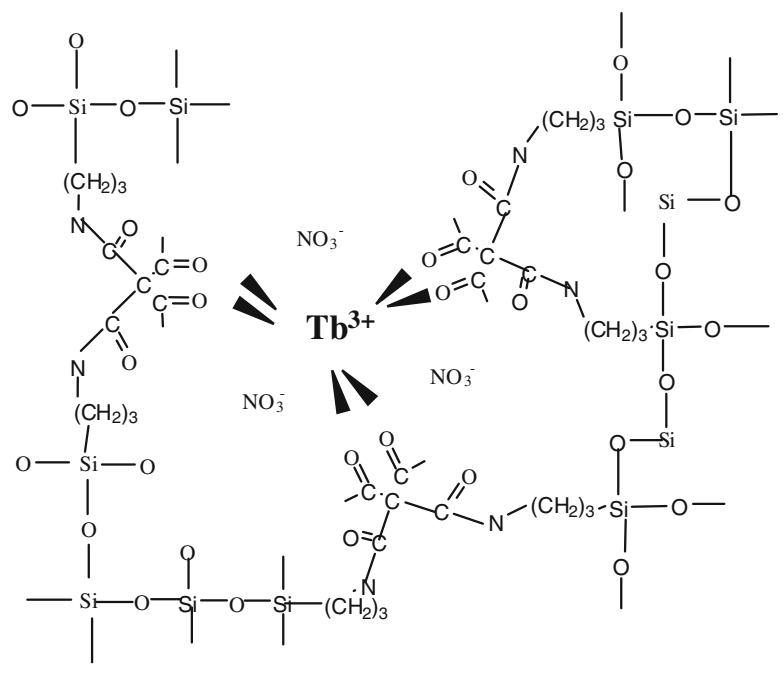

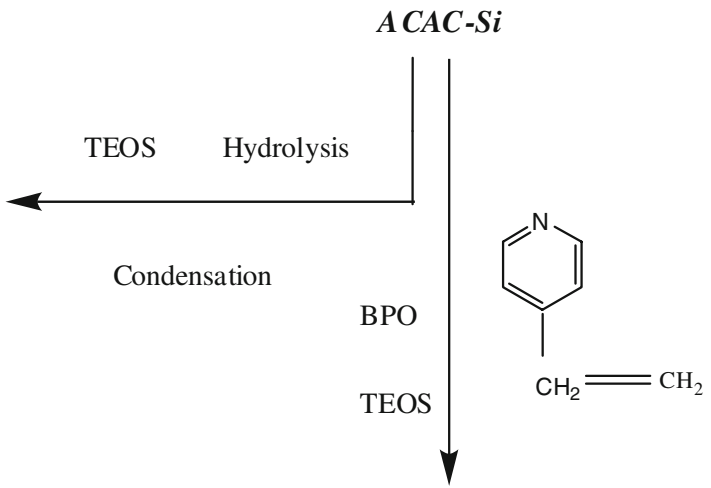

A

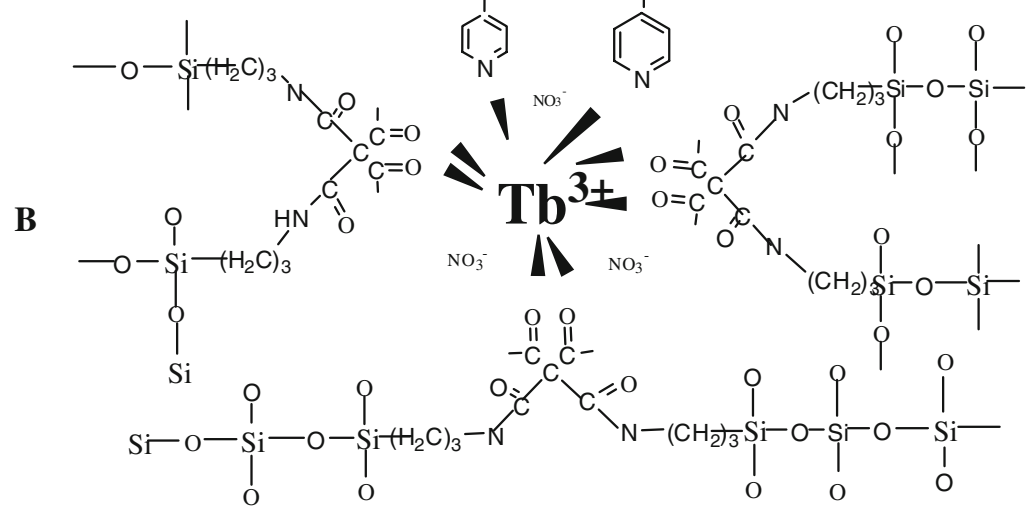

Fig. 1 Scheme of the synthesis process of ACAC-Si and predicted structure of the two hybrid materials. a the binary hybrid material and $\mathbf{b}$ the ternary hybrid material

\section{Results and discussion}

\subsection{FT-IR spectra}

Figure 2a shows the Fourier transform infrared (FTIR) spectra of the organic ligand ACAC and the precursor ACAC-Si. From the picture, the appearance of a series of strong bands at 2,973, 2,885 and $2,921 \mathrm{~cm}^{-1}$ which designated to the vibration of methylene $-\mathrm{CH}_{2}$ - suggested that the coupling reagent TEPIC was successfully grafted onto the acetylacetone. Furthermore, the bands located at $3,308 \mathrm{~cm}^{-1}$ and $1,578 \mathrm{~cm}^{-1}$ were attributed to the stretching vibration and bending vibration of grafted $-\mathrm{NH}$ group, respectively, which confirm the formation of the amide group assigned to "amide II" mode [18, 19]. Additionally, the vibration absorption around 1,165 and $1,076 \mathrm{~cm}^{-1}$ are ascribed to the stretching vibration of $\mathrm{Si}-\mathrm{C}$ and $\mathrm{Si}-\mathrm{O}$ absorption, respectively which is in accord with the successful synthesis of ACAC-Si. Figure $2 b$ and $2 c$ present the FT-IR spectra for PVP and hybrid (ACAC-Si)-Tb-PVP, respectively. From the spectra Fig. 2b, the characteristic absorption of the pyridine ring at 1596, 1550, 1492 and 1415 $\mathrm{cm}^{-1}$ can be observed obviously. Therein, $1,596 \mathrm{~cm}^{-1}$ was ascribed to the $\mathrm{C}-\mathrm{N}$ stretching vibration, and 1,550, 1,492, $1,415 \mathrm{~cm}^{-1}$ for the $\mathrm{C}-\mathrm{C}$ stretching vibration, $1,220 \mathrm{~cm}^{-1}$ for the $\mathrm{C}-\mathrm{C}$ bending vibration. While in the spectra of the hybrid (Fig. 2c), the characteristic absorption peak shift to shorter wavelength, for example, the $1,596 \mathrm{~cm}^{-1}$ for $\mathrm{C}-\mathrm{N}$ and 1,415 

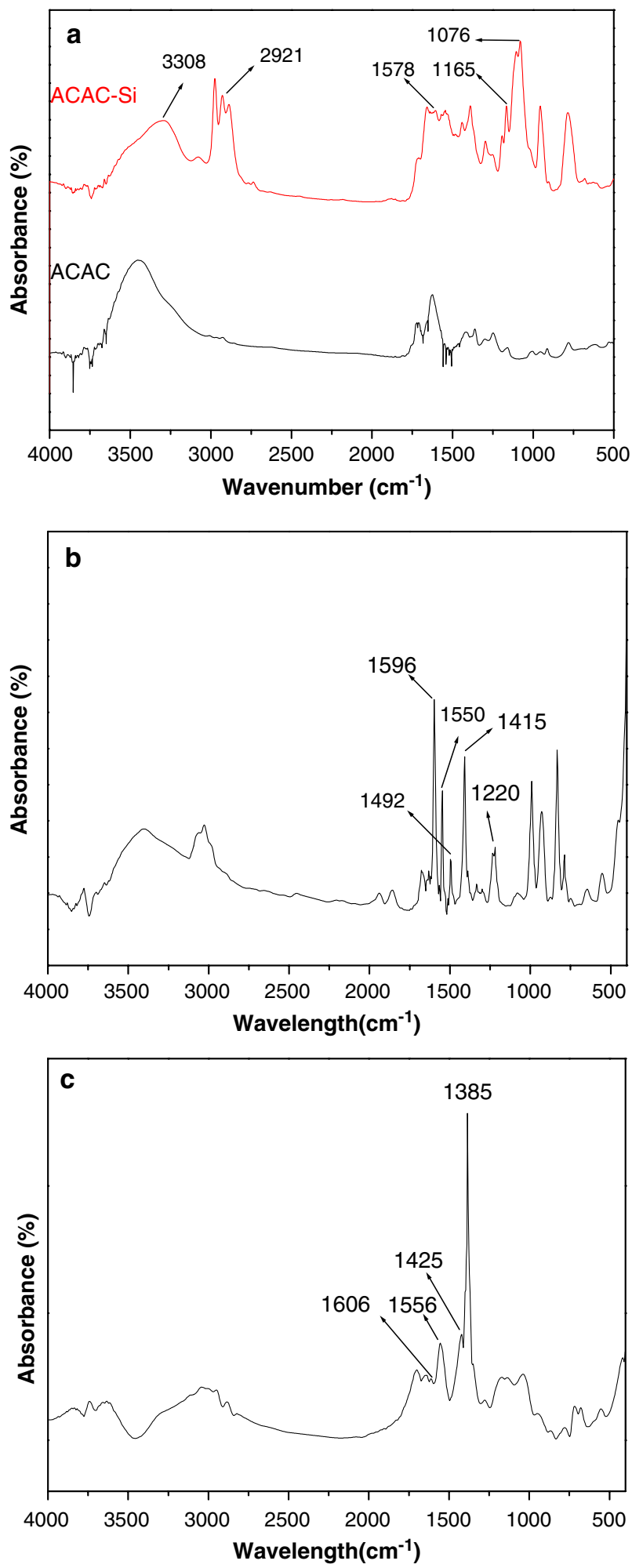

Fig. 2 IR spectra for a the free ligand ACAC and the precursor ACAC-Si; b PVP and $\mathbf{c}$ hybrid (ACAC-Si)-Tb-PVP

$\mathrm{cm}^{-1}$ for $\mathrm{C}-\mathrm{C}$ shift to 1,606 and $1,425 \mathrm{~cm}^{-1}$, respectively. This phenomenon can sustain the fact that the $\mathrm{N}$ atom of pyridine ring has coordinated with the $\mathrm{Tb}^{3+}$. Additionally, the sharp peak at $1,385 \mathrm{~cm}^{-1}$ (Fig. 2c) was considered as the $\mathrm{N}-\mathrm{O}$ stretching vibration of non-coordinated $\mathrm{NO}_{3}{ }^{-}$, and we did not see the vibration absorption of coordinated $\mathrm{NO}_{3}{ }^{-}$at $1,480,1,300,820,740 \mathrm{~cm}^{-1}$ around.

\subsection{Power XRD}

The X-ray diffraction patterns of the hybrid materials Tb-(ACAC-Si) and (ACAC-Si)-Tb-PVP unfolded in Fig. 3 reveal that both of the materials are totally amorphous. The two material exhibit similar XRD patterns, with a broad peak centered at $22.88^{\circ}$ around, which may be ascribed to the diffraction of the siliceous backbone of the hybrids. The absence of the any crystalline regions in two samples relate to the presence of organic chains in the host inorganic framework. It is worth noting that the addition of the polymeric chains makes the structure of the silica skeleton more disordered, which may result in the worse conjugating system. There are many narrow peaks in the two samples which correspond to the incomplete hydrolysiscondensation process of the excessive TEOS molecular. The excessive TEOS molecular can carry on hydrolysiscondensation reaction themselves, and then the Si-O networks form a better crystal state. Therefore, some narrow peaks appear. But most of them are very weak which indicate the content of the ordered $\mathrm{Si}-\mathrm{O}$ component is small. Moreover, it is evident that neither of the materials exhibit measurable amounts of phases corresponding to pure organic complexes and no free salt is detected too. These indications can sustain the formation of covalentbonded hybrids.

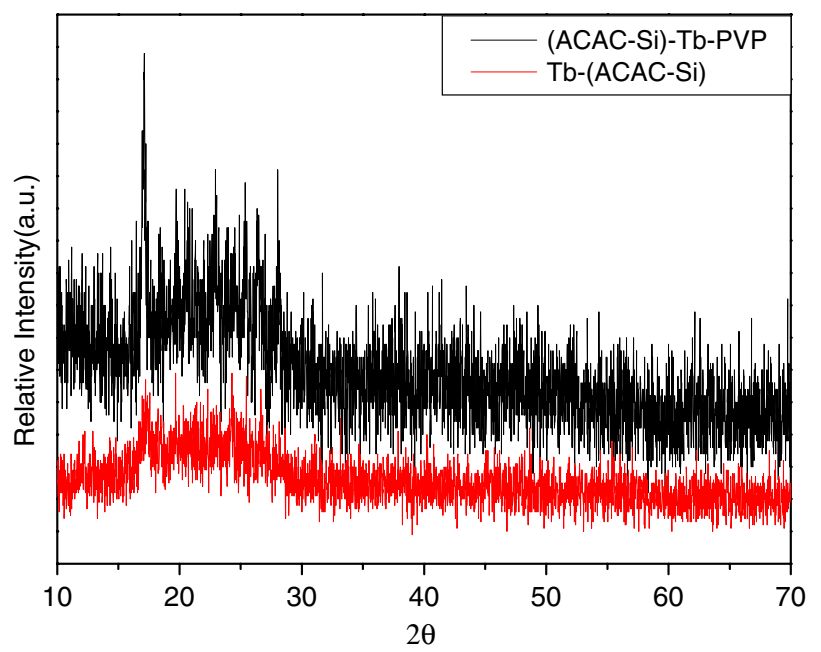

Fig. 3 X-ray diffraction (XRD) graphs of the hybrids Tb-(ACAC-Si) and (ACAC-Si)-Tb-PVP 


\subsection{Ultraviolet absorption spectra}

The ultraviolet absorption spectra of ACAC (A) and ACAC-Si (B) are shown in Fig. 4. Comparing the two spectra, a blue shift from 343 to $333 \mathrm{~nm}$ is ascribed to the major $\pi-\pi^{*}$ electronic transitions. This indicates that the electronic conjugating system of the ligand diketone was changed by the introduction of the TEPIC, which can sustain the fact that the ligand was covalently grafted to the coupling reagent and the precursor was successfully obtained in situ. Additionally, it is observed that the intensity of the absorbency has decreased which may influence the luminescent intensity of the ultimate hybrid material.

\subsection{Ultraviolet-visible diffuse reflection absorption spectra}

The ultraviolet-visible diffuse reflection absorption spectra of the hybrid materials are given in Fig. 5. The two lines denote the two hybrid materials (A for the binary hybrid material $\mathrm{Tb}-(\mathrm{ACAC}-\mathrm{Si})$ and $\mathrm{B}$ for the ternary polymeric hybrid material (ACAC-Si)-Tb-PVP, respectively). It can be seen that an inverse absorption peak located at about $545 \mathrm{~nm}$ obviously in both A and B which arises from the characteristic green emission of terbium. As shown in the picture, there exists a large broad absorption band from 220 to $600 \mathrm{~nm}$ in both curves (most of the ultraviolet visible region), resulting in the strong emission through the energy transfer system which can be observed from the fluorescence spectra. This indicates that this kind of ligand can configure large conjugated system and absorb substantive energy. According to Dexter's exchange energy transfer theory [20], the ligands absorb the energy and transfer the energy to the luminescent metal ions if the lowest triplet

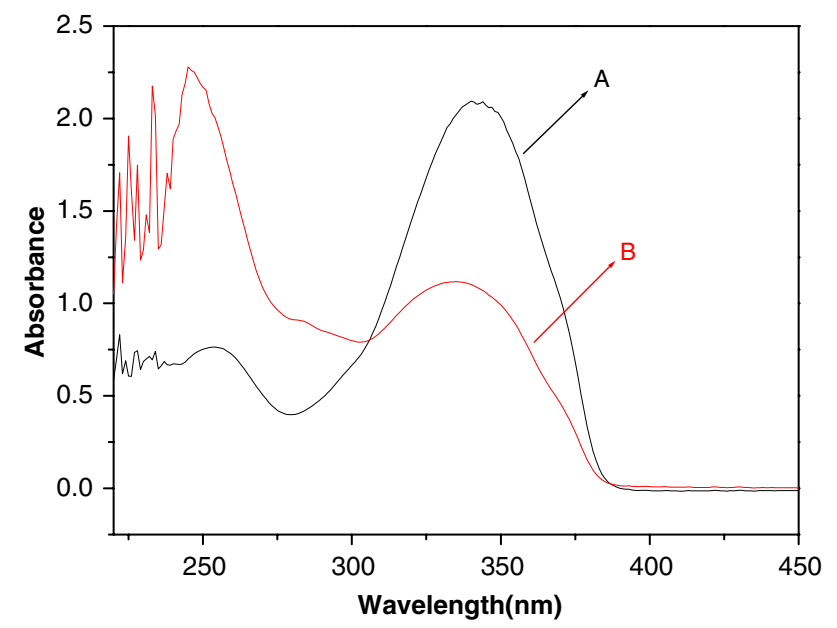

Fig. 4 UV absorption spectra of the free ligand $\operatorname{ACAC}(A)$ and the precursor ACAC-Si $(B)$

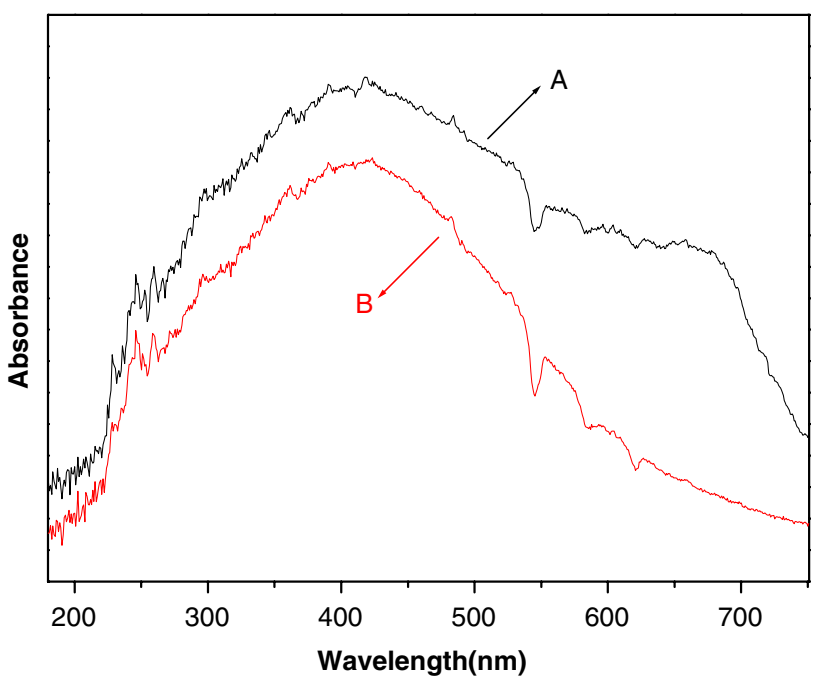

Fig. 5 The ultraviolet-visible diffuse reflection absorption spectra of the hybrid polymeric materials $\mathrm{Tb}-(\mathrm{ACAC}-\mathrm{Si})(A)$ and (ACAC-Si)Tb-PVP $(B)$

energy level of the ligands can match well with the excited energy level of the lanthanide ions. That is to say, the energy level of the ligands should be slightly higher $\left(1,500 \mathrm{~cm}^{-1}\right)$ than the resonance energy levels of the lanthanide ion [21]. It is observed that the absorption band (220-600 nm, in Fig. 3) overlaps with the excitation band (280-360 nm, in Fig. 6) which confirms that the energy difference between the ligand and the terbium ion is appropriate and this make the efficient energy transfer be possible. It is worth noting that the strong absorption in the visible region especially for $\mathrm{A}$ is ascribed to the larger delocalization of the $\pi$ electrons which accounts for a shift to the longer wavelength.

\subsection{Photoluminescence properties}

The excitation and emission spectra of the two molecular based hybrid materials Tb-(ACAC-Si) (a) and (ACAC-Si)Tb-PVP (b) are presented in Fig. 6. The excitation spectra were both obtained by monitoring the strongest emission wavelength of $\mathrm{Tb}^{3+}$ at $545 \mathrm{~nm}$. As shown in Fig. 6, the maximum absorption wavelength of the hybrids located in the range of $280-360 \mathrm{~nm} \quad(\lambda \mathrm{ex}=340 \mathrm{~nm}$ for a and $\lambda \mathrm{ex}=330 \mathrm{~nm}$ for $\mathrm{b}$ ) which is assigned to the $\pi-\pi^{*}$ transition of ligands [22, 23]. Compared with the binary hybrid material Tb-(ACAC-Si), a blue shift of the maximum excitation wavelength took place from 340 to $330 \mathrm{~nm}$ which is ascribed to the change of the surrounding coordination microenvironment of the $\mathrm{Tb}^{3+}$ ion after the introduction of the polymeric organic chain of PVP. The nitrogen atom of the vinylpyridine can also coordinated with $\mathrm{Tb}^{3+}$, but the coordination bond stability was weaker 

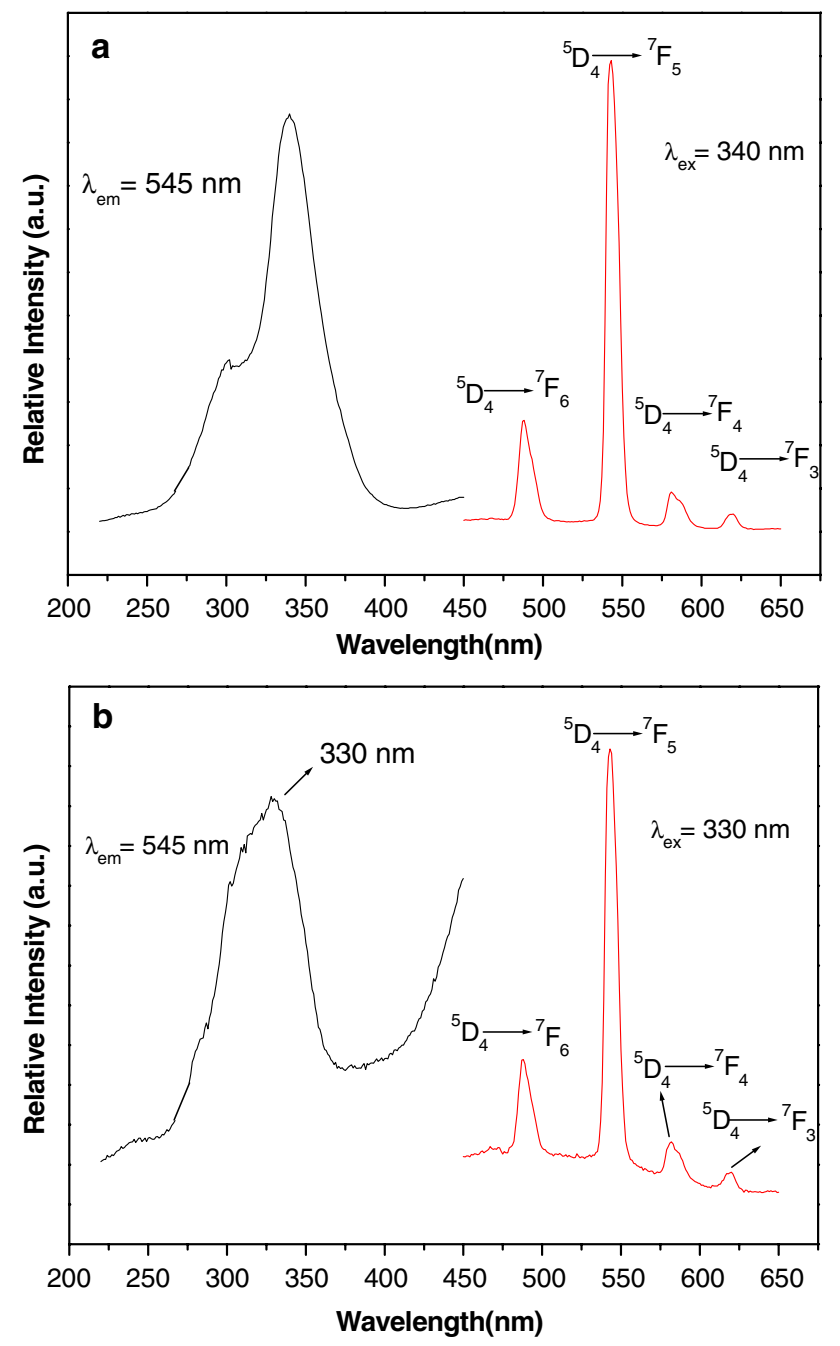

Fig. 6 The excitation and emission spectra for the hybrid materials $\mathrm{Tb}-(\mathrm{ACAC}-\mathrm{Si})$ (a) and (ACAC-Si)-Tb-PVP (b)

than oxygen, so the electronic distribution state of the primary electronic conjugating system was influenced.

From the emission spectra of the two hybrid materials, characteristic $\mathrm{Tb}^{3+}$ ion emissions are observed. It can be clearly seen that four bands in the range of $450-650 \mathrm{~nm}$ range, which are assigned to the ${ }^{5} \mathrm{D}_{4} \rightarrow{ }^{7} \mathrm{~F}_{\mathrm{J}}(\mathrm{J}=6,5,4,3)$ transitions at about $488,543,581,620 \mathrm{~nm}$, respectively. The strong green luminescence was observed in the emission spectra which indicated that the effective energy transfer took place between the modified ACAC-Si and the chelated $\mathrm{Tb}^{3+}$ ions and the triplet energy level of the ligand ACAC can match well with the resonance energy level of the $\mathrm{Tb}^{3+}$ ion which is in agreement with we mentioned above. As a result, owing to the formation of powerful chemical bonds of -Si-O-Si- backbone and the complexation of $\mathrm{Tb}^{3+}$ ( $\mathrm{Tb}-\mathrm{O}$ bond) in rare earth hybrid polymers, the hybrid polymers exhibit relatively strong emission under the unique stable chemical environment of rigid molecular network structure. Observed from Fig. 6, it is found that the green emission intensities are the strongest in all of emission of $\mathrm{Tb}^{3+}$ complexes, which can be explained by the conclusion that the leaching of the photoactive molecules was avoided and a higher concentration of rare earth ions was obtained. We can predict that ACAC is an excellent ligand which can absorb abundant energy and transfer the energy to the terbium ion, thus the addition of the polymer PVP can also make the lanthanide complex process strong luminescence emission.

\subsection{Scanning electron micrographs}

Scanning electron microscopy (SEM) was used to study the morphology of the two hybrid materials. As shown in Fig. 7, it is apparent that there is some distinction between Tb-(ACAC-Si) (a) and (ACAC-Si)-Tb-PVP (b). In Fig. 7a, it has been seen many porous pattern in the micrometer range and it seems to be very homogeneous and porous primarily due to the escape and evaporation of the solvent. We did not see the phase separation in the whole process
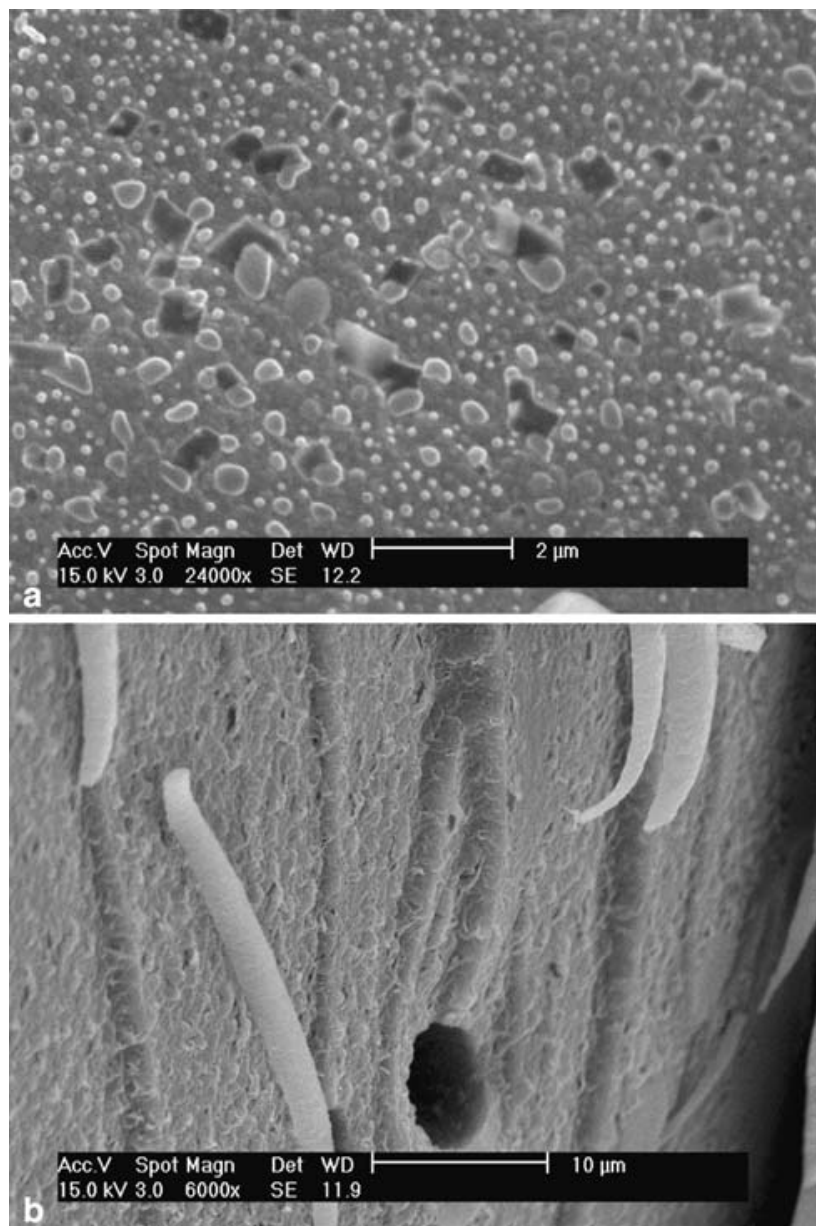

Fig. 7 SEM images of the hybrid materials Tb-(ACAC-Si) (a) and (ACAC-Si)-Tb-PVP (b) 
because of the precursor ACAC-Si acted as a functional molecular bridge linking the inorganic matrices and the organic components by covalent bonds. The hybrid material (ACAC-Si)-Tb-PVP (Fig. 7b) containing both inorganic Si-O-Si network and organic polymeric chain PVP exhibits uniform, regular and ordered microstructures with the dendritic stripe on the surface which indicates that a self-assemble process occurred during the polymerization reaction and subsequently hydrolysis and copolycondensation process.

\section{Conclusion}

In summary, we imbed the luminescent terbium complex into the polymer matrix through covalent bonds to form an organic/inorganic/polymeric hybrid material which can integrate both organic and inorganic advantages together. We have developed a novel and representative kind of synthesis and assembly method of the luminescent rareearth molecular-based polymeric hybrid materials with strong chemical bonds, which contain the long organic polymeric chains through addition polymerization and organic network (Si-O-Si) through cohydrolysis and copolycondensation reaction in sol-gel process. The homogeneous, uniform and ordered microstructure is proposed to indicate the self-assembly process occurs during the hydrolysis and polycondensation reaction. The intensive characteristic green emission of the resulting materials suggests that effective intramolecular energy transfer from the ligand to the emission of center terbium ions has taken place. We expect the organic/inorganic/polymeric hybrid materials to be potential and significant candidate in optical and electronic devices in the future.

Acknowledgments This work was supported by the National Natural Science Foundation of China (20671072) and Program for New Century Excellent Talents in University.

Open Access This article is distributed under the terms of the Creative Commons Attribution Noncommercial License which permits any noncommercial use, distribution, and reproduction in any medium, provided the original author(s) and source are credited.

\section{References}

1. G. Blasse, B.C. Grabmaier, Luminescent Materials (Springer, Berlin, 1994)

2. R. Reisfeld, C.K. Jorgensen, Laser and Excited State of Rare Earths (Springer, Berlin, 1977)

3. M. Dejneka, B. Samson, MRS Bull. 8, 39 (1999)

4. H. Mori, H. Sekiya, E. Miyoshi, K. Mogi, Y. Sakai, J. Chem. Phys. 119, 4159 (2003). doi:10.1063/1.1592505

5. A.P. Bassett, S.W. Magennis, P.B. Glover, D.J. Lewis, N. Spencer, S. Parsons, R.M. Williams, L. De Cola, Z. Pikramenou, J. Am. Chem. Soc. 126, 9413 (2004). doi:10.1021/ja048022z

6. A.I. Voloshin, N.M. Shavaleev, V.P. Kazakov, J. Lumin. 93, 115 (2001). doi:10.1016/S0022-2313(01)00177-6

7. M.R. Robinson, M.B. O'Regan, G.C. Bazan, Chem. Commun. (Camb.) 17, 1645 (2000). doi:10.1039/b004739m

8. C.Y. Yang, V. Srdanov, M.R. Robinson, G.C. Bazan, A.J. Heeger, Adv. Mater. 14, 980 (2002)

9. K. Kuriki, Y. Koike, Y. Okamoto, Chem. Rev. 102, 2347 (2002). doi:10.1021/cr010309g

10. A.M. Klonkowski, S. Lis, M. Pietraszkiewicz, Z. Hnatejko, K. Czarnobaj, M. Elbanowski, Chem. Mater. 15, 656 (2003). doi: $10.1021 / \mathrm{cm} 0202557$

11. P.A. Tanner, B. Yan, H.J. Zhang, J. Mater. Sci. 35, 4325 (2000). doi:10.1023/A:1004892520502

12. N.I. Koslova, B. Viana, C. Sanchez, J. Mater. Chem. 3, 111 (1993). doi:10.1039/jm9930300111

13. P. Huhtinen, M. Kivelä, O. Kuronen, V. Hagren, H. Takalo, H. Tenhu, T. Lövgren, H. Härmä, Anal. Chem. 77, 2643 (2005). doi:10.1021/ac048360i

14. Q.M. Wang, B. Yan, Inorg. Chem. Commun. 7, 1124 (2004). doi: 10.1016/j.inoche.2004.08.015

15. Q.M. Wang, B. Yan, J. Photochem. Photobiol. Chem. 177, 1 (2006). doi:10.1016/j.jphotochem.2005.02.021

16. Q.M. Wang, B. Yan, J. Photochem. Photobiol. Chem. 175, 159 (2005). doi:10.1016/j.jphotochem.2005.04.030

17. B. Yan, F.F. Wang, J. Organomet. Chem. 692, 2395 (2007). doi: 10.1016/j.jorganchem.2007.02.025

18. M.M. Coleman, K.H. Lee, D.J. Skrovanek, P.C. Painter, Macromolecules 19, 2149 (1986). doi:10.1021/ma00162a008

19. L.S. Fu, R.A. Sá Ferreira, N.J.O. Silva, L.D. Carlos, Chem. Mater. 16, 1507 (2004). doi:10.1021/cm035028z

20. D.L. Dexter, J. Chem. Phys. 21, 836 (1953). doi:10.1063/1. 1699044

21. R.V. Deun, P. Fias, P. Nockemann, K.V. Hecke, L.V. Meervelt, K. Binnemans, Inorg. Chem. 45, 10416 (2006). doi:10.1021/ic06 16277

22. W.H. Zhang, X.B. Lu, J.H. Xiu, Z.L. Hua, L.X. Zhang, M. Robertson, J.L. Shi, D.S. Yan, J.D. Holmes, Adv. Funct. Mater. 14, 544 (2004). doi:10.1002/adfm.200305001

23. J.G. Bünzli, E. Moret, V. Foiret, K.J. Schenk, M. Wang, L. Jin, J. Alloys Compds. 107, 207 (1994) 